\title{
Irrigation et intensification de la production agricole au Sahel : perceptions paysannes sur la double riziculture et les variétés de riz utilisées sur les aménagements hydro - agricoles au Niger
}

\author{
Hamadou S. ${ }^{\text {* }}$, Mossi Maïga, I. ${ }^{2}$, Kanfidéni, B. ${ }^{3}$, \\ 1 Union Économique et Monétaire Ouest Africaine (UEMOA) \\ 2 Université de Tillabér, BP : 175 Tillabéri-Niger \\ 3 Institut National de la Recherche Agronomique du Niger (INRAN). B.P. 429 Niamey Niger \\ * Correspondance : Seyni HAMADOU, Union Économique et Monétaire Ouest Africaine, 01 B.P.454 Ouagadougou 01, \\ Burkina Faso. Tél : (226) 76654392 / 700060 53, Fax : (226) 253188 72, \\ E-mail : hseyni@yahoo.com \\ Original submitted in on $1^{\text {st }}$ September 2016. Published online at www.m.elewa.org on $30^{\text {th }}$ November 2016 \\ http://dx.doi.org/10.4314/jab.v107i1.9
}

\section{RÉSUMÉ}

Objectif : L'étude vise à cerner les perceptions paysannes sur la double riziculture et les variétés utilisées au Niger.

Méthodologie et Résultats: Elle a été menée sous forme d'enquête auprès de 345 producteurs de 10 aménagements hydro-agricoles de la vallée du fleuve Niger. Malgré le caractère aléatoire de l'agriculture pluviale, le riz demeure une culture de second rang tandis que la maîtrise de l'eau à tendance à favoriser le non-respect du calendrier cultural engendrant une augmentation des coûts de production et une baisse des rendements. La double riziculture se caractérise par une dizaine de goulots d'étranglement $(P<0,01)$ dont 3 apparaissent majeures : la contrainte de main d'œuvre, l'étiage du fleuve et l'approvisionnement en engrais. Sept sources de fourniture de semences ont été identifiées tandis que près de trente contraintes sont signalées sur les variétés $(P<0,001)$. Les plus importantes sont la faible résistance aux maladies, l'exigence des variétés en engrais et le faible niveau de rendement

Conclusion et application des résultats : L'état de la filière riz au Niger mérite la réhabilitation des services d'encadrement des producteurs, la mise en place d'un système efficace d'approvisionnement en intrants de qualité et une intensification de la recherche en vue de la mise au point de variétés à la fois résistantes aux maladies et moins exigeantes en engrais.

Mots clés : Agriculture irriguée, perceptions paysannes, double riziculture, variétés de riz 


\title{
Hamadou et al., J. Appl. Biosci. 2016. Irrigation et intensification de la production agricole au Sahel : perceptions paysannes sur la double riziculture au Niger \\ Irrigation and intensification of agricultural production in the Sahel: peasant perceptions on dual rice cultivation and rice varieties used in irrigation schemes in Niger
}

\begin{abstract}
Objective: The study aims to determine farmer's perceptions on modern rice farming system and varieties used by farmers

Methodology and Results: The study was carried out form a survey near 345 producers from 10 hydroagricultural perimeters of the Niger River valley. In spite of the overdrawn character of rain agriculture in Niger, rice remains a second-rate culture while the water control supports the non-observance of the farming calendar, which generates an increase in the loads of production and a fall of the yields. Double rice production is characterized by ten constraints $(P<0.01)$ of which 3 appear major: the labour force unavailability, low water level of the river and inputs provisioning. According to varieties used, seven providing sources was identified and nearly thirty constraints are announced $(P<0.001)$. Most important are low diseases resistance, fertilizers high requirement and yield low level.

Conclusion and application of results : Niger rice production system situation require the rehabilitation of farmers framing services, the installation of a good quality inputs provisioning mechanism and an the intensification of the research in the field of the development of varieties at the same time resistant to the diseases and less demanding in fertilizer.
\end{abstract}

Key words: Irrigated agriculture, farmer's perceptions, rice annual double production, rice varieties

\section{INTRODUCTION}

Face à la croissance démographique soutenue (3,3\% par an) et à la récurrence des déficits pluviométriques depuis la fin des années 1960, le développement de l'irrigation est devenue une priorité pour l'ensemble des gouvernements qui se sont succédés au Niger (République du Niger, 1987a, République du Niger, 2012a, République du Niger, 2012b). D'importants investissements ont été consentis pour aménager plus de 87 Aménagements Hydro - Agricoles (AHA) dont 40 sont exclusivement destinés à la double riziculture (saison sèche et saison d'hivernage), soit environ 15000 ha de riz par an avec maîtrise totale de l'eau (République du Niger, 2015). Pour soutenir la filière moderne du riz, une ferme de semences de riz a été créée et les producteurs, organisés en coopératives, sont chargés de la mise en valeur des périmètres, de la gestion de l'eau, de l'approvisionnement en engrais et de la commercialisation primaire du paddy. Les prix au producteur sont garantis tandis que les prix des engrais sont subventionnés, la taxe des importations de riz relevée et l'office public de commercialisation du riz, Riz du Niger (RINI), doté d'un monopole à l'achat et à l'usinage du paddy. Le système ainsi mis en place a fait passer la production de moins de 5000 tonnes de paddy au début des années 1970 à plus de 82000 tonnes aujourd'hui (République du Niger, 2014). II faut cependant noter que le système a connu des difficultés au lendemain du séminaire tenu à Zinder en 1982. Ce forum national était annonciateur de l'application prochaine d'un Programme d'Ajustement Structurel (PAS) - le pays y a souscrit quatre années plus tard - qui a préconisé le désengagement de l'État des différents secteurs de production, le transfert de la gestion des AHA aux coopératives et la suppression de la subvention aux intrants (République du Niger, 1997). Depuis, la filière est confrontée à d'énormes difficultés : les coopératives, les processus de gestion et les procédures de contrôle mis en place après 1982 se sont révélés incapables d'assurer la maintenance et le renouvellement des équipements hydrauliques (République du Niger, 2000). La pérennité des aménagements, qui bénéficient aux familles de plus de 30000 exploitants, est remise en cause (République du Niger, 2015). L'objectif de cette étude était d'appréhender les perceptions des producteurs sur la double riziculture et sur les variétés qui leurs sont proposées. Elle se veut une contribution à l'atténuation des goulots d'étranglement de la filière riz au Niger. 


\section{MATÉRIEL ET MÉTHODES}

Zone d'étude : L'étude a été réalisée dans la vallée du fleuve Niger, qui concentre plus de $75 \%$ des périmètres aménagés et exclusivement destinés à la riziculture au Niger (Figure 2). Elle s'étend sur les zones agroécologiques, sud sahélienne où la pluviométrie varie entre $300 \mathrm{~mm}$ et $700 \mathrm{~mm}$ et, soudanienne semi-humide où la pluviométrie oscille entre 700 à $800 \mathrm{~mm}$ (République du Niger, 1998). Les températures varient entre $16^{\circ} \mathrm{C}$ et $22^{\circ} \mathrm{C}$ en décembre-janvier et entre $27^{\circ} \mathrm{C}$ et $40^{\circ} \mathrm{C}$ en mars-avril.

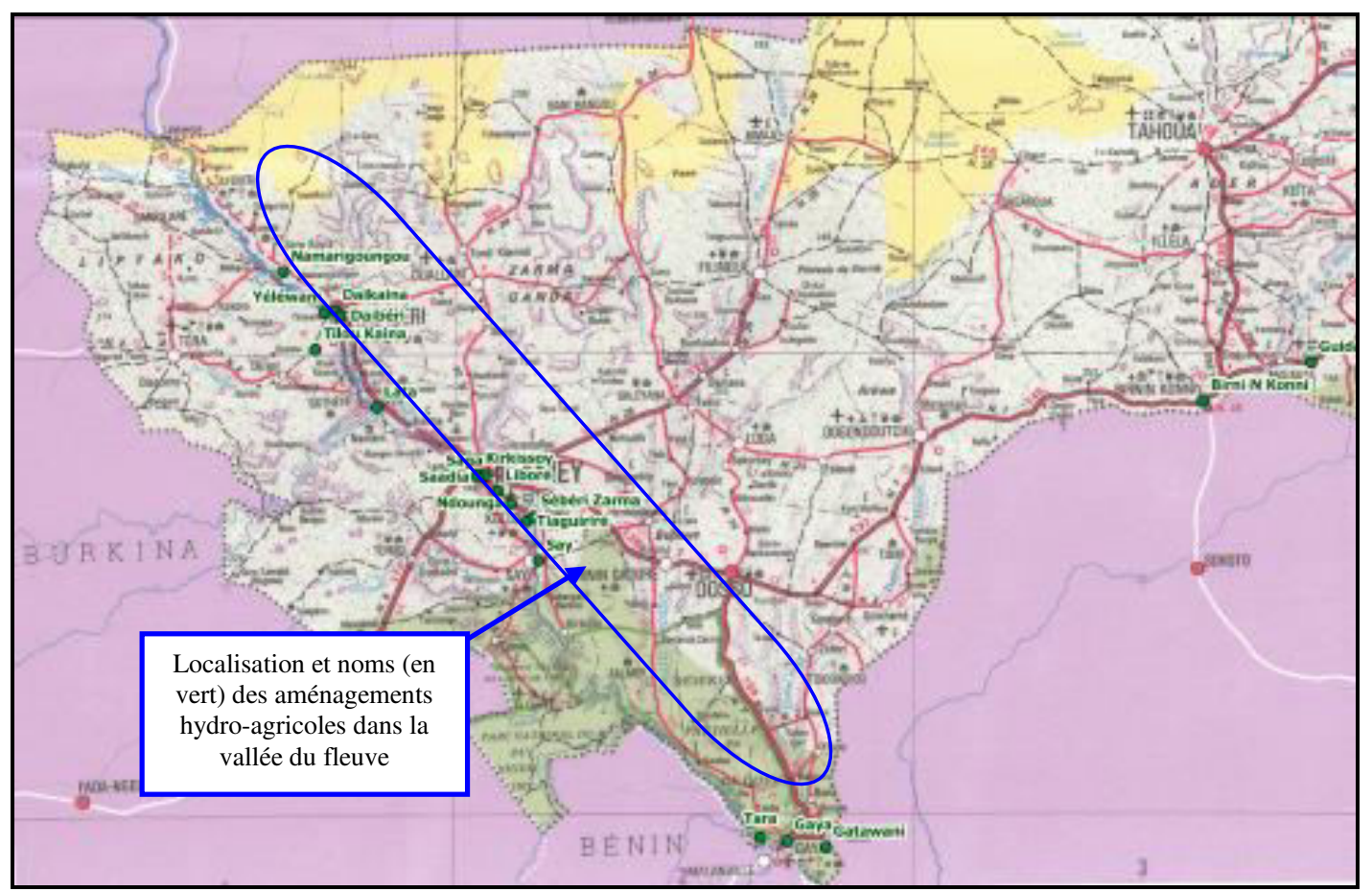

Figure 2 : localisation des aménagements hydro-agricoles dans la vallée du fleuve

Source : République du Niger, 2011

Les systèmes de production du riz dans la vallée du fleuve Niger au Niger: Trois grands systèmes de production de riz sont pratiqués dans notre zone d'étude (République du Niger, 2000). La riziculture traditionnelle. Elle est sous inondation et se pratique essentiellement en bordure du fleuve ou dans les mares pendant l'hivernage d'où, sa forte dépendante vis à vis des crues et de la pluie. Elle occupe environ 10000 ha avec des rendements de 0,7 tonnes/ha (République du Niger, 2005). La riziculture irriguée sur AHA public. Elle est à maîtrise totale de l'eau, ce qui permet une double production dans l'année. Ce système assure $83 \%$ de la production du pays, soit environ 82000 tonnes de paddy par an (République du Niger, 2015). Malgré le contexte intensif de la production, le travail est essentiellement manuel à l'exception des labours qui se font en culture attelée. La petite irrigation privée. Elle porte le plus souvent sur de petites exploitations sur aménagement sommaire cultivant le riz en hivernage et le maraîchage en saison sèche. Les surfaces sont estimées à 1500 ha et les rendements moyens autour de 3 tonnes/ha (République du Niger, 2005).

Collecte des données: L'étude est basée sur une enquête de terrain auprès d'un échantillon de producteurs dont le choix a été raisonné. Dans un premier temps, les AHA ont été subdivisés en 3 groupes : périmètres urbains pour les AHA situés dans la capitale Niamey et ses environs immédiats, frontaliers pour les périmètres situés dans la zone de Gaya, région la plus humide du Niger et à cheval entre le Niger, le Nigeria et le Bénin et, ruraux pour tous les autres périmètres. Cette classification part du constat des problèmes organisationnels dissemblables sur ces trois catégories de périmètres. Sur les AHA urbains où beaucoup de fonctionnaires y sont recensés, les périmètres sont relativement mieux exploités et l'approvisionnement en engrais se pose avec moins d'acuité qu'en milieu rural et dans la zone de Gaya. En outre, les AHA de la zone de Gaya sont des périmètres de deuxième génération, c'est-à-dire, créés après 1992 où les difficultés de la gestion des grands aménagements 
par les producteurs ont amené les pouvoirs publics à geler la construction de nouveaux périmètres, « conforter " les périmètres existants et favoriser le développement de la « petite irrigation privée » plutôt que la création de nouveaux périmètres collectifs. Dans une seconde étape, nous avons fixé la base de sondage des AHA à $25 \%$ des périmètres, soit 10 périmètres sur la base des $40 \mathrm{AHA}$ de la vallée du fleuve Niger exploités par 4856 producteurs. Ils ont été tirés de façon aléatoire mais pondérée en fonction du nombre total de périmètres dans chaque catégorie. En troisième lieu, nous avons fixé le taux de

\section{RÉSULTATS}

Caractéristiques des exploitations et place du riz dans le système de production: Les informations relatives aux 10 AHA étudiés sont présentées dans le Tableau 1. Sur les 486 exploitants retenus, 345 ont effectivement pris part à l'enquête, soit $71 \%$. Parmi eux, nous avons recensé seulement $2,6 \%$ de femmes : $4,8 \%$ sondage des producteurs à $10 \%$ des exploitants de chaque périmètre retenu, tirés de façon aléatoire. Les données ont été collectées sur la base d'un questionnaire structuré en quatre parties : caractéristiques structurales des exploitations, perceptions paysannes sur les variétés cultivées et la double riziculture, production et impact de la riziculture à l'échelle des exploitations. Une fois les différentes modalités codifiées et les données saisies, le traitement des informations générées a été basé sur des tests statistiques classiques, calcul de fréquence et test de khi-deux réalisés à l'aide du logiciel SPSS.

des exploitants des périmètres urbains et 2,3\% des exploitants des AHA ruraux. Les paramètres démographiques n'indiquent pas de grandes variations entre les exploitations (Tableau 2) de même que l'expérience des chefs d'exploitation en matière de production rizicole.

Tableau $1:$ Informations sur les sites d'enquêtes et taille de l'échantillon

\begin{tabular}{llcccccc}
\hline AHA & Type & Latitude Nord & $\begin{array}{c}\text { Longitude } \\
\text { Est }\end{array}$ & $\begin{array}{c}\text { Création } \\
\text { Superficie } \\
\text { (ha) }\end{array}$ & $\begin{array}{c}\text { Exploitants a } \\
\text { Échantillon } \\
\text { (N) }\end{array}$ \\
\hline Saga & Urbain & $1^{\circ} 28^{\prime} 06^{\prime \prime}$ & $2^{\circ} 09^{\prime} 00^{\prime \prime}$ & 1966 & 395 & 1081 & 69 \\
Sadia Aval & Urbain & $1^{\circ} 30^{\prime} 54^{\prime \prime}$ & $2^{\circ} 07^{\prime} 12^{\prime \prime}$ & 1985 & 26 & 55 & 15 \\
Daibéri & Rural & $1^{\circ} 10^{\prime} 02^{\prime \prime}$ & $1^{\circ} 29^{\prime} 55^{\prime \prime}$ & 1986 & 350 & 572 & 70 \\
Sébéri & Rural & $1^{\circ} 17^{\prime} 56^{\prime \prime}$ & $2^{\circ} 20^{\prime} 57^{\prime \prime}$ & 1979 & 397 & 1100 & 44 \\
Karaïgorou & Rural & $1^{\circ} 32^{\prime} 55^{\prime \prime}$ & $1^{\circ} 59^{\prime} 56^{\prime \prime}$ & 1970 & 144 & 437 & 36 \\
Say 1 & Rural & $1^{\circ} 06^{\prime} 28^{\prime \prime}$ & $2^{\circ} 21^{\prime} 46^{\prime \prime}$ & 1981 & 250 & 354 & 37 \\
Gaya Amont & Frontalier & $1^{\circ} 53^{\prime} 19^{\prime \prime}$ & $3^{\circ} 29^{\prime} 30^{\prime \prime}$ & 1990 & 170 & 325 & 13 \\
Tara & Frontalier & $1^{\circ} 54^{\prime} 01^{\prime \prime}$ & $3^{\circ} 20^{\prime} 15^{\prime \prime}$ & 1975 & 120 & 572 & 7 \\
Kessa & Frontalier & $1^{\circ} 54^{\prime} 01^{\prime \prime}$ & $3^{\circ} 20^{\prime} 15^{\prime \prime}$ & 1994 & 45 & 180 & 20 \\
Sékondji Birni & Frontalier & $1^{\circ} 50^{\prime} 47^{\prime \prime}$ & $3^{\circ} 36^{\prime} 24^{\prime \prime}$ & 1994 & 45 & 180 & 34 \\
\hline
\end{tabular}

a Nombre d'exploitants au moment de la création

La taille des exploitations varie d'un minimum de 3 ha en milieu urbain à 5 ha en zone frontalière et 9 ha en milieu rural. Les spéculations y sont nombreuses, en particulier en milieu rural. En termes de superficie, le riz occupe la 4 ème place en milieu rurbain $(0,7 \mathrm{ha})$ contre la $6{ }^{\mathrm{ème}}$ et 7 ème place respectivement en région frontalière $(0,4 \mathrm{ha})$ et zone rurale $(0,5 \mathrm{ha})$. Malgré l'étroitesse de la taille des exploitations en milieu urbain, le riz est majoritairement perçu comme culture principale $(57,1 \%$ des exploitants). Dans les deux autres zones, cette place est dévolue au mil $(51,7 \%$ des exploitants des périmètres frontaliers et $47 \%$ des exploitants des périmètres ruraux). 


\begin{tabular}{l}
$\begin{array}{l}\text { Hamadou et al., J. Appl. Biosci. 2016. Irrigation et intensification de la production agricole au Sahel: } \\
\text { perceptions paysannes sur la double riziculture au Niger }\end{array}$ \\
\hline
\end{tabular}

Tableau 2 : Caractéristiques structurales des exploitations

\begin{tabular}{|c|c|c|c|c|}
\hline Paramètres & Urbain & Frontalier & \multicolumn{2}{|c|}{ Rural } \\
\hline Age CE a & $49(10)$ & $47(11)$ & \multicolumn{2}{|c|}{$49(11)$} \\
\hline Population b & $10(4)$ & $14(13)$ & \multicolumn{2}{|c|}{$11(6)$} \\
\hline Actif $\mathrm{c}$ & $5(3)^{\prime}$ & $7(6)$ & \multicolumn{2}{|c|}{$6(4)$} \\
\hline Expérience ${ }^{d}$ & $26(10)$ & $19(12)$ & \multicolumn{2}{|c|}{$24(12)$} \\
\hline Animaux détenus & & & & \\
\hline Ânes & $1(0)$ & $1(0)$ & \multicolumn{2}{|l|}{$2(3)$} \\
\hline Bovins & $3(3)$ & $3(1)$ & \multicolumn{2}{|l|}{$6(9)$} \\
\hline Ovins & $4(2)$ & $5(7)$ & \multicolumn{2}{|c|}{$6(11)$} \\
\hline Caprins & $5(3)$ & $3(3)$ & \multicolumn{2}{|c|}{$6(14)$} \\
\hline Chevaux & - & - & $3(2)$ & \\
\hline Chameaux & - & - & $2(-)$ & \\
\hline Équipements & & & & \\
\hline Charrette asine & $1(0)$ & $1(0)$ & $1(0)$ & \\
\hline Charrette bovine & $1(0)$ & $1(0)$ & $1(1)$ & \\
\hline Faucille & $1(-)$ & $3(2)$ & $3(1)$ & \\
\hline Charrue & $1(0)$ & $1(0)$ & $1(0)$ & \\
\hline Motopompe & & $1(0)$ & $1(1)$ & \\
\hline Herse & $1(-)$ & $1(-)$ & & \\
\hline Exploitation (ha) e & $2,6(4,5)$ & $4,7(5,4)$ & $7,2(6,3$ & \\
\hline Cultures $^{f}$ & & & & \\
\hline Arbres fruitiers & - & $0,1(-) \quad[2,1]$ & - & \\
\hline Manioc & - & $0,3(0,3) \quad[6,3]$ & - & \\
\hline Piment & - & $0,25(-) \quad[5,8]$ & - & \\
\hline Coton & & $-[0,0]$ & $0,3(-)$ & {$[5,8]$} \\
\hline Arachide & $0,5(0,4) \quad[10,5]$ & - & $0,5(0,3)$ & {$[5,7]$} \\
\hline Sésame & - & - & $0,4(0,5)$ & {$[4,3]$} \\
\hline Gombo & - & - & $0,3(0,3)$ & {$[3,5]$} \\
\hline Jardin & - & - & $0,4(0,3)$ & {$[4,2]$} \\
\hline Mais & $0,6(0,6)$ & - & $1,6(2,1)$ & [18] \\
\hline Mil & $0,8(0,7) \quad[17,9]$ & $2,6(2,3) \quad[53,7]$ & $2,8(3,2)$ & [31] \\
\hline Niébé & $0,3(0,3) \quad[6,5]$ & $0,9(0,9) \quad[19,8]$ & $0,7(0,6)$ & {$[7,5]$} \\
\hline Oignon & $0,3(0) \quad[5,8]$ & - & $0,2(0,0)$ & {$[2,7]$} \\
\hline Oseille & $0,1(0)$ & - & $0,2(-)$ & {$[1,7]$} \\
\hline Riz & $0,7(1,3) \quad[15,9]$ & $0,4(0,6) \quad[8,6]$ & $0,5(0,8)$ & {$[5,4]$} \\
\hline Sorgho & $0,7(0,9) \quad[15,4]$ & $0,5(0,3) \quad[9,5]$ & $1,3(1,5)$ & [14] \\
\hline Tomate & - & - & $0,2(-)$ & {$[2,2]$} \\
\hline
\end{tabular}

${ }^{a}$ Age du chef d'exploitation (années) ; b Taille de la famille (personnes), ${ }^{c}$ Nombre de personnes d'un âge compris entre 15 et 65 ans; d Expérience en matière de culture de riz (années) ; e Taille de l'exploitation (ha), ${ }^{\mathrm{f}}$ Surface occupée par les différentes spéculations (ha) ; Les chiffres entre parenthèses représentent les écarts - type

Les chiffres entre crochets représentent les parts relatives (\%) occupées en termes de superficie.

En ville, $12 \%$ des exploitants considèrent le riz comme aliment de base et $9,1 \%$ le considèrent comme principale source de revenu. En milieu rural, $7,3 \%$ des exploitants le considèrent comme aliment de base et $63 \%$ comme principale source de revenu. Dans la région la plus arrosée du Niger, il est l'aliment de base de seulement $1,2 \%$ des exploitants et, considéré comme principale source de revenu par moins de $9 \%$ des exploitants.
Perceptions paysannes sur la double riziculture : Sur les AHA enquêtés, $31 \%$ des producteurs estiment la double riziculture contraignante $(P<0,0001)$. Par type d'aménagement, ces statistiques s'établissent à $74,1 \%$ sur les périmètres frontaliers, $34,3 \%$ sur les périmètres ruraux et seulement $9,5 \%$ sur les périmètres urbains. La double riziculture se caractérise par une dizaine de 


\begin{tabular}{l}
$\begin{array}{l}\text { Hamadou et al., J. Appl. Biosci. 2016. Irrigation et intensification de la production agricole au Sahel : } \\
\text { perceptions paysannes sur la double riziculture au Niger }\end{array}$ \\
\hline
\end{tabular}

contraintes $(P<0,01)$ dont, 3 apparaissent majeures (Tableau 3) :

1. La disponibilité de la main d'œuvre (33\% des riziculteurs : $50 \%$ des producteurs urbains, $32,1 \%$ des producteurs des périmètres frontaliers et $34,7 \%$ des producteurs ruraux).
2. L'étiage du fleuve se traduisant par des difficultés d'irrigation en certaines périodes $(27,7 \%$ des riziculteurs : $25 \%$ des producteurs urbains, $39,3 \%$ des producteurs frontaliers et $24,8 \%$ des producteurs ruraux). 3. L'approvisionnement en engrais $(22,6 \%$ des riziculteurs : $17,9 \%$ des riziculteurs des AHA frontaliers et $25,7 \%$ des ruraux).

Tableau 3 : Perceptions paysannes et principales contraintes de la double riziculture

\begin{tabular}{lcccc}
\hline & Urbain & Frontalier & Rural & Total \\
\hline Existence de contraintes $(\%)$ & $8(9,5 \%)$ & $20(74,1)$ & $69(34,3)$ & $97(31,1)$ \\
\hline \multicolumn{1}{c}{ Khi deux $\left(\chi^{2}\right)=42,505$} & \multicolumn{2}{c}{ Degré de Liberté $=2$} & \multicolumn{2}{c}{$\mathrm{P}<0,0001$} \\
\hline Problème de main d'œuvre en hivernage & $4(50)$ & $9(32,1)$ & $35(34,7)$ & $48(35)$ \\
Inondation en hivernage & - & - & $5(4,9)$ & $5(3,7)$ \\
Attaques d'oiseaux en hivernage & - & - & $2(1,9)$ & $2(1,5)$ \\
Maladies en hivernage & $2(25)$ & - & $1(0,9)$ & $3(2,2)$ \\
Problème d'eau en saison sèche & $2(25)$ & $11(39,3)$ & $25(24,8)$ & $38(27,7)$ \\
Difficulté de production des plants & - & - & $2(1,9)$ & $2(1,5)$ \\
Approvisionnement en engrais & - & $5(17,9)$ & $26(25,7)$ & $31(22,6)$ \\
Fertilité sol & - & $1(3,6)$ & $2(1,9)$ & $3(2,2)$ \\
Problème d'équipement & - & $2(7,1)$ & $1(0,9)$ & $3(2,2)$ \\
Écoulement du paddy & - & - & $2(1,9)$ & $2(1,5)$ \\
Total & $8(100)$ & $28(100)$ & $101(100)$ & $137(100)$ \\
\hline \multicolumn{1}{c}{ Khi deux $\left(\chi^{2}\right)=33,42$} & \multicolumn{2}{c}{ Degré de Liberté = 18 } & $\mathrm{P}<0,05$ & \\
\hline
\end{tabular}

Perceptions paysannes sur les variétés utilisées: Plusieurs variétés sont rencontrées sur les AHA et il n'a pas été possible de retrouver l'appellation d'origine, les paysans ayant donné des noms locaux à certaines. L'enquête révèle en effet, plusieurs sources d'approvisionnement en semences : 3 pour les producteurs des périmètres urbains $(50 \%$ au marché, $30 \%$ sur le périmètre et $20 \%$ à la ferme de production de semences), 7 pour les producteurs des périmètres frontaliers $(32,26 \%$ au marché, $29,14 \%$ au village, $19,35 \%$ sur le périmètre, $9,68 \%$ au service agricole d'arrondissement et, $3,23 \%$ respectivement à la ferme de production de semences, au Bénin et au Nigeria,) et, 7 sur les périmètres ruraux $(31,65 \%$ sur le périmètre,
$34,18 \%$ au marché, $20,25 \%$ au village, $8,86 \%$ à la ferme, $2,53 \%$ au Nigeria, $1,27 \%$ respectivement au Bénin et au service agricole d'arrondissement). Dans le traitement des données, seules les variétés contenues dans le catalogue national des semences (République du Niger, 2012c) ont été considérées. II s'agit de : IR 15 29, IR 22, BG 90 2, D 52 37, Sintane Diofor, Guiza 175, Wita 8 et Wita 9. Plusieurs contraintes sont relevées par les producteurs en ce qui concerne les variétés qu'ils utilisent $(P<0,0001)$. En milieu urbain, les contraintes identifiées sont au nombre de 18 (Tableau 4) dont les plus importantes sont la faible résistance aux maladies $(32,9 \%)$, le faible niveau de rendement $(17,9 \%)$, les avortements en saison sèche (13\%) et la verse (10\%). 


\begin{tabular}{l}
$\begin{array}{l}\text { Hamadou et al., J. Appl. Biosci. 2016. Irrigation et intensification de la production agricole au Sahel : } \\
\text { perceptions paysannes sur la double riziculture au Niger }\end{array}$ \\
\hline
\end{tabular}

Tableau 4 : Perceptions des producteurs des AHA de type urbain sur les variétés de riz cultivées

\begin{tabular}{|c|c|c|c|c|c|c|c|c|}
\hline Contraintes & BG 902 & D 5237 & Guiza 175 & IR 1529 & IR 8 & Wita 8 & Wita 9 & Total \\
\hline Faible résistance aux maladies & 41 & 3 & 1 & 15 & 4 & 2 & 2 & 68 \\
\hline Faible rendement & 3 & 15 & 1 & 5 & 1 & 8 & 4 & 37 \\
\hline Avortement en SS & & & & & 1 & 26 & & 27 \\
\hline Verse & & & 16 & 1 & 1 & 2 & 1 & 21 \\
\hline Difficulté reprise plants âgés & & & & & 1 & 7 & & 8 \\
\hline Faible résistance à la chaleur & & & & 1 & & 6 & & 7 \\
\hline Brisure & & & & 1 & & 3 & & 4 \\
\hline Maturité étalée & & & & & & & 4 & 4 \\
\hline Plat pâteux & & & & & 4 & & & 4 \\
\hline Récolte difficile & & & 4 & & & & & 4 \\
\hline Faible tallage & & & & 2 & & 1 & & 3 \\
\hline Trop de paille & & & 3 & & & & & 3 \\
\hline Chaumes trop courts & & & & & 1 & & 1 & 2 \\
\hline Cycle trop court & & & & & 2 & & & 2 \\
\hline $\begin{array}{l}\text { Faible résistance mauvaises } \\
\text { herbes }\end{array}$ & & & & & 1 & & 1 & 2 \\
\hline Exigeante en engrais & & & 1 & & & & & 1 \\
\hline Non adapté AHA & & 1 & & & & & & 1 \\
\hline Non adapté parcelle & & & & 1 & & & & 1 \\
\hline Total & 45 & 22 & 26 & 26 & 20 & 55 & 13 & 207 \\
\hline$x^{2}=1543,0$ & & Degré & le Liberté $=$ & 140 & $\mathrm{P}<$ & 0,0001 & & \\
\hline
\end{tabular}

En zone frontalière, les contraintes identifiées sont au nombre de 14 (Tableau 5) dont les plus fréquentes sont la faible résistance aux maladies $(26,4 \%)$, la faible résistance aux mauvaises herbes (19,5\%), l'exigence des variétés en engrais $(12,6 \%)$ et la faible résistance à la sécheresse $(12,6 \%)$.

Tableau 5 : Perceptions des producteurs des AHA de type frontalier sur les variétés de riz cultivées

\begin{tabular}{|c|c|c|c|c|c|c|c|c|}
\hline Contraintes & BG 902 & D 5237 & Guiza 175 & IR 1529 & IR 8 & Wita 8 & Wita 9 & Total \\
\hline Faible Résistance aux maladies & & & & 16 & 6 & 1 & & 23 \\
\hline $\begin{array}{l}\text { Faible Résistance mauvaises } \\
\text { herbes }\end{array}$ & & & & 4 & 13 & & & 17 \\
\hline Exigeante en engrais & & & & 7 & 4 & & & 11 \\
\hline Faible Résistance Sécheresse & & & & 4 & 6 & 1 & & 11 \\
\hline Faible tallage & & & & 4 & & 1 & & 5 \\
\hline Difficulté reprise plants âgés & & & & 1 & 3 & & & 4 \\
\hline Faible Résistance inondation & & & & 1 & 3 & & & 4 \\
\hline Cycle trop long & & 1 & & 1 & & & & 2 \\
\hline Difficulté de commercialisation & & 1 & & & & 1 & & 2 \\
\hline Faible rendement & & & & & & 1 & 1 & 2 \\
\hline Faible Résistance au froid & & & & 2 & & & & 2 \\
\hline Verse & & & & & 2 & & & 2 \\
\hline Avortement en SS & & & & & & 1 & & 1 \\
\hline Nécessite hautes eaux & & 1 & & & & & & 1 \\
\hline Total & & 3 & & 43 & 37 & 7 & 1 & 87 \\
\hline Khi deux $\left(\chi^{2}\right)=1392,973$ & & Degré de & iberté $=70$ & & & & $P<0,0$ & \\
\hline
\end{tabular}

En milieu rural, les contraintes sont au nombre de 24 (Tableau 7), dont les plus fréquentes sont la faible résistance aux maladies $(36,1 \%)$, le faible niveau des rendements $(17,5 \%)$, le mauvais goût $(7,2 \%)$, la faible 


\begin{tabular}{l}
$\begin{array}{l}\text { Hamadou et al., J. Appl. Biosci. 2016. Irrigation et intensification de la production agricole au Sahel : } \\
\text { perceptions paysannes sur la double riziculture au Niger }\end{array}$ \\
\hline
\end{tabular}

résistance à la sécheresse $(6,5 \%)$, la faible résistance variétés en engrais $(5,2 \%)$.

aux attaques des insectes (5,5\%) et l'exigence des

Tableau 7 : Perceptions des producteurs des AHA de type rural sur les variétés de riz cultivées

\begin{tabular}{|c|c|c|c|c|c|c|c|c|}
\hline Contraintes & BG 902 & D 5237 & $\begin{array}{c}\text { Guiza } \\
175\end{array}$ & IR 1529 & IR 8 & Wita 8 & Wita 9 & Total \\
\hline $\begin{array}{l}\text { Faible résistance aux } \\
\text { maladies }\end{array}$ & 39 & & & 43 & 3 & 13 & 7 & 105 \\
\hline Faible rendement & 8 & 5 & & 8 & & 22 & 8 & 51 \\
\hline Mauvais goût & 4 & 3 & & & 10 & 1 & 3 & 21 \\
\hline $\begin{array}{l}\text { Faible résistance à la } \\
\text { sécheresse }\end{array}$ & 5 & 1 & 1 & & 2 & 9 & 1 & 19 \\
\hline $\begin{array}{l}\text { Faible résistance aux } \\
\text { insectes }\end{array}$ & 6 & & & 4 & & 2 & 4 & 16 \\
\hline Exigeante en engrais & & 1 & & 13 & & 1 & & 15 \\
\hline Avortement en SS & & & & & & 11 & 1 & 12 \\
\hline Brisure & & & & 1 & & 11 & & 12 \\
\hline Graines trop légères & & & & 2 & & 6 & 2 & 10 \\
\hline Faible tallage & 1 & 1 & & & & 3 & 2 & 7 \\
\hline Difficulté reprise plants âgés & & & & 6 & & & & 6 \\
\hline Verse & & & & & & 3 & & 3 \\
\hline Chaumes trop courts & & & & 1 & & & 1 & 2 \\
\hline $\begin{array}{l}\text { Difficulté de } \\
\text { commercialisation }\end{array}$ & & 1 & & & & 1 & & 2 \\
\hline Battage difficile & & & & 1 & & & & 1 \\
\hline Besoin bon planage & & & & & & 1 & & 1 \\
\hline Besoin sol profond & & & & & & 1 & & 1 \\
\hline Chaumes trop fragiles & & & & & & 1 & & 1 \\
\hline Faible résistance à la chaleur & & & & & & 1 & & 1 \\
\hline Faible résistance aux & & 1 & & & & & & 1 \\
\hline mauvaises herbes & & & & & & & & \\
\hline Grains trop petits & & & & & & & 1 & 1 \\
\hline Nécessite hautes eaux & & 1 & & & & & & 1 \\
\hline Non adapté AHA & & 1 & & & & & & 1 \\
\hline Trop de paille & 1 & & & & & & & 1 \\
\hline Total & 64 & 15 & 1 & 86 & 15 & 89 & 33 & 291 \\
\hline \multicolumn{2}{|c|}{ Khi deux $\left(\chi^{2}\right)=2179,332$} & \multicolumn{3}{|c|}{$\mathrm{DL}=175$} & \multicolumn{4}{|c|}{$p<0,0001$} \\
\hline
\end{tabular}

\section{DISCUSSION}

L'examen de la place du riz dans le système de production montre, malgré le caractère déficitaire de la production agricole, que le riz demeure une culture de second rang par rapport au mil tandis que l'importance qui lui est accordée est marginale en zone de relative sécurité alimentaire comme c'est le cas de la zone frontalière. Contrairement à nos attentes, nos résultats montrent que la commercialisation du paddy se pose avec moins d'acuité alors même que la concurrence du riz importé a toujours été mise en avant pour expliquer les difficultés des filières rizicoles en Afrique en général et au
Niger en particulier (Benz et Hassane, 1995, Hirsch, 1998; Aw, 1998). II est d'ailleurs spécifique aux périmètres ruraux où $1,9 \%$ des riziculteurs déclarent $y$ être confrontés. L'inondation des parcelles en hivernage est spécifique aux périmètres ruraux et concerne $5 \%$ des producteurs. S'il est vrai qu'elle peut révéler un défaut dans la conception des AHA concernés ou un déficit d'entretien des drains, son importance mérite d'être connue car elle doit déterminer le choix des variétés sur les superficies concernées. II en est de même de la difficulté de production des plants qui peut résulter d'un 
problème de qualité de semences mais également du non-respect du calendrier cultural. Au plan du double riziculture, la première contrainte se rapporte à la main d'œuvre. Elle est particulièrement forte en saison d'hivernage où une dualité apparaît dans la répartition de la main d'œuvre entre les champs dunaires et le riz, en milieu rural notamment. Nos enquêtes montrent que pendant l'hivernage, le repiquage du riz est fréquemment reporté jusqu'à la fin du semis des cultures pluviales et, il est fréquent de voir des paysans repiquer du riz en épiaison pendant cette campagne. Tout se passe comme si la maîtrise de l'eau engendrait le non-respect du calendrier cultural dont les conséquences sont l'augmentation des charges de production essentiellement due à la fourniture d'eau aux retardataires et la baisse des rendements. Des travaux antérieurs (République du Niger, 1998 ; Anders et al. 1984; Maïkoréma, 1986 ; Rassas et Loute, 1989) ont montré que pendant la saison des pluies, les riziculteurs de la vallée du fleuve Niger négligent le riz au profit des autres cultures, en particulier le mil. D'après Yu et al. (1994), cette situation qui se traduit par l'allocation prioritaire de la main d'œuvre familiale à l'accroissement des rendements du mil plutôt qu'à la maximisation du revenu, résulterait du fait que la valeur marginale de l'utilisation de la main d'œuvre est plus importante pour le mil que pour le riz, en particulier durant la période de soudure allant du début de l'hivernage à la récolte. Durant cette période, les stocks de la campagne écoulée sont épuisés alors même que les besoins alimentaires sont plus importants du fait des travaux champêtres. En ce qui concerne le problème d'eau, il est lié en partie au non respect du calendrier cultural proposé par l'Office National des Aménagements Hydro-Agricoles (ONAHA). Il se décompose en une campagne de saison sèche (SS) allant du 15 novembre au 25 mai et une campagne de saison d'hivernage (SH) allant du 15 juin au 25 décembre. II a été déterminé pour combiner les contraintes climatiques (influence de la température sur le développement et la fécondation du riz) avec celles relatives aux disponibilités en eau du fleuve Niger (Adamou et al., 2002). En conséquence, tout décalage par rapport à ce calendrier a des répercutions sur les rendements de la culture et sur les conditions de fonctionnement des pompes. Cette dernière contrainte est particulièrement forte pour les périmètres dont les chenaux d'alimentation en eau des pompes ont un débit limitant en période d'étiage avec risque de cavitation et de dégradation des pompes (République du Niger, 2000). C'est le cas, par exemple, de l'ensemble des périmètres frontaliers dont la taille relativement réduite a milité pour l'installation de motopompes diesel plutôt qu'électrique. Quant à la difficulté d'approvisionnement en engrais, aucun producteur ne la relève en milieu urbain contre $25,7 \%$ des exploitants des périmètres ruraux et $17,9 \%$ des exploitants des périmètres frontaliers. Cette situation s'explique par le manque de solvabilité des producteurs et des coopératives des périmètres ruraux qui empêche les livraisons d'intrants à crédit par les opérateurs privés. Sur les périmètres frontaliers, la faiblesse de la proportion des producteurs s'explique par la proximité du Bénin et du Nigeria qui facilite l'accessibilité aux intrants du fait notamment des disparités des politiques macroéconomiques (Hamadou, 1997). Au plan des variétés, le premier goulot réside dans les difficultés d'approvisionnement en semences nées de l'effondrement du système d'approvisionnement mis en place (Schalbroeck et Djib, 1999). Nos enquêtes montrent qu'avec l'autonomie conférée aux coopératives, la procédure d'approvisionnement établie n'est pas suivie et dans $50 \%$ voire $90 \%$ des cas, nous ne pouvons pas parler de semences de qualité, mais plutôt de tout-venant avec parfois, des "semences " qui franchissent les frontières sans aucun contrôle. D'autre part, quel que soit le type de périmètre considéré, la faible résistance du riz aux maladies apparait comme la principale contrainte sur les AHA au Niger. Aucune variété n'est épargnée même si la variété IR 1529 semble la plus affectée. II s'agit probablement du virus de la panachure jaune du riz (Rice yellow mottle virus, RYMV) qui est devenue la principale maladie de la riziculture en Afrique de l'Ouest. Au Niger, les pertes dues à cette maladie sont estimées entre $58 \%$ et $68 \%$ de la production. La RYMV peut donc être la cause de la faiblesse des rendements, mais également, des avortements signalés par les producteurs. Cependant, le fait que les avortements soient signalés pendant la campagne dite de saison sèche milite en faveur du non respect du calendrier cultural qui prolonge le cycle de production au point où, la floraison intervient en pleine chaleur. L'exigence des variétés en engrais constitue également une contrainte majeure relevée par les producteurs. Elle est spécifique aux périmètres ruraux et frontaliers et peut témoigner de la mauvaise qualité des engrais qui fait que les producteurs qui ne respectent généralement pas les doses préconisées $(200 \mathrm{~kg} / \mathrm{ha}$ d'engrais composé NPK (15-15-15) après le repiquage et $200 \mathrm{~kg} / \mathrm{ha}$ d'urée à la reprise, $200 \mathrm{~kg} / \mathrm{ha}$ d'urée au tallage et $50 \mathrm{~kg} / \mathrm{ha}$ d'urée à l'initiation paniculaire) constatent une mauvaise réponse des plants aux engrais. La faible performance des plants de riz face à la concurrence des mauvaises herbes est spécifique à la zone humide tandis que la faible résistance des variétés à la sécheresse 
concerne les périmètres ruraux et frontaliers. L'ensemble de ces contraintes et en particulier la faible résistance aux mauvaises herbes traduit le besoin de variétés spécifiques adaptées à la zone. Enfin, nous retiendrons

\section{CONCLUSION ET IMPLICATIONS}

Jadis modèle de référence dans la sous région Ouestafricaine, la double riziculture nigérienne est confrontée à d'énormes difficultés depuis le désengagement de l'État qui a engendré un effondrement des services d'encadrement des producteurs. L'étude révèle que les contraintes de la riziculture sont tout aussi importantes à l'amont qu'à l'aval de la production mais surtout, présentent des spécificités en fonction de la localisation géographiques des périmètres. La première spécificité réside dans la perception des producteurs sur l'importance du riz dans le système de production. L'étude révèle le peu d'engouement à la production du riz lorsque la sécurité alimentaire des populations n'est pas menacée mais également, l'influence négative de la maîtrise de l'eau au niveau des paysans non ou malsensibilisés. La seconde spécificité réside dans les perceptions paysannes sur les contraintes de la double riziculture. Alors que la majorité des études imputent les difficultés de la filière essentiellement à la concurrence du riz importé, notre étude montre que ce problème est

\section{RÉFÉRENCES BIBLIOGRAPHIQUES}

Adamou, H., Hamadou, S., Kupper, A.et Kupper, M., 2002. La disponibilité en eau du fleuve Niger: enjeux et perspectives pour les Aménagements Hydro - Agricoles (AHA). Le cas du Niger. Les monographies sahéliennes $n^{\circ} 13$. Bamako : INSAH - AGROSOC - GRN - SA; $79 p$.

ADRAO, 2000. Rapport annuel ADRAO 2000 : points saillants des activités. M'Bé, ADRAO. 37p.

Anders, G., Firestone, W., Gould, M., Malek, E., Simmons, E., Versel, M., Ware, T. and Zalla, T., 1984. Niger irrigation subsector assessment. Volumes 1 and 2, Niamey, USAID. 32p.

Aw, D., 1998. Analyse de la filière riz : Niger. Accra. FAO - Programme de coopération technique, $58 \mathrm{p}$.

Benz, H., Fusillier J.-L., Hassane, S., Liagre, Mendez del Villar J., Sourrisseau M. 1995. Les premiers effets de la dévaluation sur les filières riz irrigués au Sahel (Sénégal, Mali, Niger). Synthèse. Montpellier, CIRAD/Ministère de la Coopération, $51 \mathrm{p}$.

Hamadou, S., 1997. Libéralisation du commerce des produits vivriers au Niger et mode d'organisation d'autres contraintes comme le goût et la verse recensées respectivement sur les périmètres urbains et ruraux témoignant de l'utilisation des semences tout-venant.

secondaire et concerne moins de $2 \%$ des producteurs. Les principaux goulots d'étranglement résident au niveau de la disponibilité en main d'œuvre, de la disponibilité en eau du fleuve Niger, l'approvisionnement en engrais spécifique aux périmètres ruraux et frontaliers et, l'approvisionnement en semences de qualité incertaine dans $50 \%$ à plus de $90 \%$ des sources d'approvisionnement. La filière riz au Niger mérite, compte tenu du niveau d'intensification recherché, que les services d'encadrement des producteurs soient réhabilités et mieux appuyés dans les missions qui sont les leurs. Un système efficace d'approvisionnement en intrants agricole (engrais et semences) de qualité doit être mis en place. La recherche doit s'orienter vers la mise au point de variétés à la fois productives, résistantes aux maladies, au stress hydrique et à la verse, moins exigeantes en engrais. Par ailleurs, le problème lié à la rareté et donc la cherté de la main d'œuvre nécessite que la riziculture au Niger soit mécanisée dans ses différentes activités.

des acteurs privés. Les réseaux marchands dans le fonctionnement du système de commercialisation des céréales. Thèse de doctorat. Montpellier : ENSAM, 348p.

Hirsch, R. D., 1998. La riziculture dans les pays de I'UEMOA : de la dévaluation à la libéralisation 1993-1997. Paris, AFD $59 p+$ annexes.

Maïkoréma, Z. B., 1986. Gestion de deux systèmes de culture dans les exploitations agricoles du fleuve Niger. Rennes : École Nationale Supérieure Agronomique de Rennes, Chaire d'Économie Rurale. $78 \mathrm{p}$.

Rassas, B. et Loute, T., 1989. Rice and cotton Policy. Technical Report No 106 (Draft). Niamey, USAID. 30p

République du Niger, 2015. Rapport annuel ONAHA 2015. Niamey, ONAHA. 48p.

République du Niger, 2012a. Initiative 3N pour la sécurité et le développement agricole durable: " les Nigériens nourrissent les nigériens ». Cadre stratégique. Vision 2035 et plan d'action 2011 - 
2015, Niamey, Haut-commissariat à l'Initiative 3 N. $87 p$.

République du Niger, 2012b. Plan de développement économique et social du Niger 2012 - 2015. Ministère du Plan, de l'aménagement du territoire et du développement communautaire. Niamey, 156p.

République du Niger, 2012c. Catalogue national des espèces et variétés végétales (CNEV) : le riz. Niamey, FAO. 39p

République du Niger, 2011. Étude complémentaire de faisabilité du Programme "Fonds de réhabilitation des périmètres publics irrigués ", Niamey, MAG-ABN-Kfw, 214p.

République du Niger, 2005. Stratégie nationale du développement de l'irrigation et des eaux de ruissellement (SNDI/CER). Niamey, Ministère du développement rural, $84 \mathrm{p}$.

République du Niger, 2003. Stratégie de développement rural : le secteur rural, principal moteur de la croissance économique, Niamey, Cabinet du Premier Ministre, 65p.

République du Niger, 2000. Programme d'appui à la filière riz : étude de faisabilité. Rapport provisoire. Niamey, CIRAD-Ministère du Plan, $64 p+$ annexes.

République du Niger, 1997. Revue du secteur rural du Niger. Niamey, MAG-DEP-PASA. 167p + annexes.

République du Niger, 1987a. Plan de développement économique et social du Niger 1987 - 1991 : Présentation résumée. Ministère du Plan, Niamey. 48p.

Schalbroeck, J. - J. et Djibo, N., 1999. Projet semencier/ONAHA : rapport d'activité 1998 1999. Niamey, MAG/EL - ONAHA. 72p.

Yu, Z., Deuson, R., Bomans, E. et Lowemberg-DoBoer, J. 1994. Analysis of the competition for labor by dryland and irrigated crops: the case of rice and millet in Niger. Tucson: Journal for farming Systems research-extension ; (4) 2: 27-44.

Zaslavsky J., Dabat M-H., Nguyen G., Bosc P-M., Hamadou S., Lidon B., Mossi Maïga I., 2000, Programme d'appui à la filière riz: étude de faisabilité, Niamey, Union EuropéenneRépublique du Niger.72p. 